\section{Multimodal pediatric pain management (part 2)}

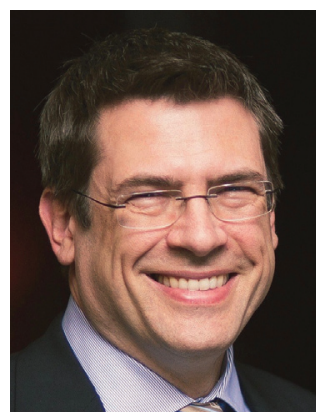

Dr Stefan Friedrichsdorf* speaks to Commissioning Editor Jade Parker: Stefan Friedrichsdorf, MD, is medical director of the Department of Pain Medicine, Palliative Care and Integrative Medicine at Children's Hospitals and Clinics of Minnesota in Minneapolis/St Paul, MN, USA, home to one of the largest and most comprehensive programs of its kind in the country. The pain and palliative care program is devoted to control acute, chronic/complex and procedural pain for inpatients and outpatients in close collaboration with all pediatric subspecialties at Children's Minnesota. The team also provides holistic, interdisciplinary care for children and teens with life limiting or terminal diseases and their families. Integrative medicine provides and teaches integrative, nonpharmacological therapies (such as massage, acupuncture/acupressure, biofeedback, aromatherapy and selfhypnosis) to provide care that promotes optimal health and supports the highest level of functioning in all individual children's activities. In this second part of the interview they discuss multimodal (opioid-sparing) analgesia for hospitalized children in pain and how analgesics and adjuvant medications, interventions, rehabilitation, psychological and integrative therapies act synergistically for more effective pediatric pain control with fewer side effects than a single analgesic or modality.

First draft submitted: 14 November 2016; Accepted for publication: 14 December 2016; Published online: 20 January 2017

Q Children's Minnesota specializes in treating babies, children \& teenagers. In your view, when you strongly advocate for multimodal analgesia, what are the most effective strategies for the treating these different age groups of patients?

The underlying pathophysiology of pain in children may include acute nociceptive pain (i.e., pain arising from the activation of peripheral nerve endings, including somatic and visceral pain), neuropathic pain (i.e., resulting from injury to, or dysfunction of, the somatosensory system), psycho-social-spiritual-emotional pain, total pain, and/ or chronic persistent pain (i.e., pain extending beyond the expected time of healing). Pain in hospitalized children may originate from one, but more commonly involves a combination of these pathophysiologies. The best practice of preventing and treating pain in children involves using multimodal (opioid-sparing) analgesia, which may include analgesics and adjuvants, procedural interventions, rehabilitation, psychological and integrative therapies that act synergistically for more effective pediatric pain control with fewer side effects than any single analgesic or modality [1].

*Department of Pain Medicine, Palliative Care \& Integrative Medicine, Children's Hospitals \& Clinics of Minnesota, Minneapolis, MN, USA

and

Department of Pediatrics, University of Minnesota, Minneapolis, MN, USA

Stefan.Friedrichsdorf@childrensmn.org

\section{Pain Management}

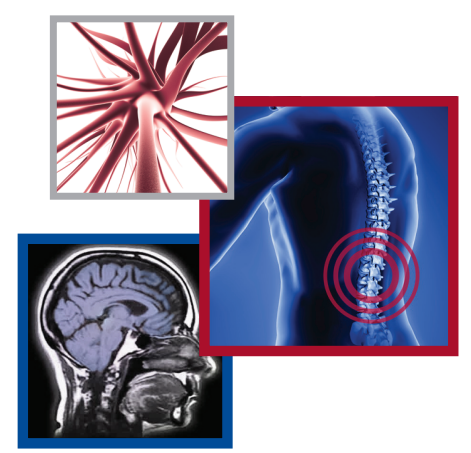

KEYWORDS

- analgesia • integrative strategies

- multimodal $•$ pediatric pain management 
Key to the effective use of a multimodal approach is that we are not simply treating according to the pain score anymore. In other words, a higher pain number does not necessarily equal more morphine administration. Understanding a child's pain depends on the whole clinical picture. How much pain we perceive on a scale of $0-10$ depends on how dangerous our brain perceives this pain to be. For example, a child may be very distracted by playing a video game and when we look at the child we assume the patient could not be in pain, when in fact it is the distraction that helps to downregulate the nociception. That explains why analgesia appears to be better throughout the daytime when there also are plenty of distractors, whereas at bedtime and during the night distress seem to escalate.

Multimodal analgesia may include basic analgesics, such as paracetamol/acetaminophen and ibuprofen; opioids, such as morphine, fentanyl, hydromorphone, oxycodone or methadone; and/or adjuvant analgesics, such as gabapentinoids, $\alpha$-agonists, low-dose tricyclics, NMDA channel blockers and nerve blocks or neuroaxial anesthesia. But pain is increased by stress, anxiety, depression, catastrophizing, school absenteeism, poor sleep hygiene, among others; and for a complex pain situation, pharmacology alone is often insufficient. Depending on the clinical scenario using physical therapy and exercise (especially when children are physically deconditioned), psychology, implementing sleep hygiene, returning to school and normalizing social life commonly are the most effective ways to normalize function and reduce and eliminate pain. Integrative, nonpharmacological modalities, which we commonly integrate into the treatment plan include breathing strategies, aromatherapy, biofeedback, progressive muscle relaxation, autogenic training, mindfulness, yoga and/or my favorite modality: self-hypnosis [2].

If there are no clinical signs of acute tissue injury, and pain continues beyond the expected time of healing (which is how we define chronic pain) then in fact we have seen that opioids are not beneficial and under no circumstances would we then prescribe morphine or other opioids. In children with primary pain disorders, such as primary headaches, centrally mediated abdominal pain syndrome or widespread musculoskeletal pain, we help patients to go back to their normal life as soon as possible. Primary pain disorders also co-exist, or can even be triggered by underlying organic disease, and pain symptoms do not necessarily represent inadequate treatment, flare-up or recurrence.

\section{Q Does the pain treatment vary with} condition, age, gender or personality type? Pain prevention and treatment varies with age and the cognitive and developmental level of the individual infant, toddler, school child or adolescent. In addition the pharmacodynamics and pharmacokinetics of analgesic medication is altered in infants younger than 3-6 months, and as a result metabolize medications differently than older children. The younger an infant is, the lower the starting dose for opioids are for treating acute pain. However, the flip side of neuroplasticity is development of rapid tolerance, which means that babies very quickly get tolerant to opioids and therefore we have to titrate the dosage faster than with older children. We also know that morphine is metabolized by the liver into a 'good guy' (analgesic 40-100-times more effective than morphine) called morphine6-glucuronide (M6G) and the 'bad guy' (nociceptive) morphine-6-glucuronide (M3G). The latter accumulates in infants at a much higher rate than in children or teenagers. This means that in premature infants, morphine often does not work for acute pain procedures and therefore should not be a first-line medication in this case.

When we think about other modalities, clearly physical therapy, exercise, active integrative modalities, hypnosis for a toddler would look very different than that for an active 17 years old. So we need to be flexible when looking at the activity and cognitive level of the patient, and all our modalities clearly have to be appropriate for this age group as well as the individual patient. We might use biofeedback for older children, whereas for younger children we might prefer using music and massage. In terms of mind-body techniques, for younger children the parents often are the caregivers and we might teach them appropriate behavioral interventions they can implement to help the children, whereas we expect older patients to practice the modalities themselves.

\section{Q When your team is seeing infants \&} children in pain: what are the most common underlying pathophysiologies?

In the chronic outpatient pain clinic the most common are:

- Primary headaches, such as tension headaches and migraines; 
- Centrally mediated abdominal pain syndrome (which used to be called 'functional abdominal pain');

- Widespread musculoskeletal and joint pain (formerly called 'juvenile fibromyalgia');

- Complex regional pain syndrome type 1;

- Coexisting chronic-on-acute pain: children with underlying recurrent organic pain conditions such as inflammatory bowel disease including Crohn's disease, juvenile rheumatoid arthritis, status post ventricular-peritoneal shunt, sickle cell disease, vascular necrosis, among others. These are children who commonly have overcome their acute tissue injury, but remain in pain beyond their expected time of healing.

In the inpatient pain service the most common are:

- Postoperative pain;

- Cancer pain stemming from either the malignancy itself or as a result of the cancer treatment, for example, chemotherapy-induced mucositis;

- Sickle cell disease;

- Episodes of inconsolability. For example an infant who is inconsolable, we have to explore jointly with the primary team whether it is pain (as oppose to delirium, withdrawal, 'neuro-irritability' or medication side effect, among others), and then what type of pain it might be. Examples include visceral pain, acute nociceptive pain, neuropathic pain, psycho-social-spiritual pain and so on. A large number of children with complex medical needs might develop a feeding intolerance or visceral hyperalgesia, and feeding through a tube eventually might cause physical discomfort. In addition, a significant number of children especially on intensive care units who may look like they are in pain, might be in fact delirious in part as a result of too much or inappropriate pain and sedation medication.

\section{Q Chronic pain in children is common,} complex \& complicated, right? What does research show in explaining why some children become very dysfunctional despite normal clinical workup?

One of the highest stressors for many clinicians in a children's hospital or pediatric clinic in fact might be taking care of this surprisingly large number of children (and their parents) who develop chronic pain. The interesting thing is, if we look into the research, that a large number of children and teenagers at one time or another experience prolonged pain. [3] The three most common pain disorders are headaches or migraines, abdominal pain and joint pain or muscle pain. The vast majority of these kids do not become dysfunctional, they experience this pain episode and they just go on with their normal life [4]. However, it seems to be that about $4-5 \%$ of those kids who experience pain frequently can become dysfunctional, which means that they start missing school and experience insomnia among other things. What research has shown us is that it is actually not one single thing that causes the chronic pain and dysfunction, but that it really is a large number of individual pieces of straw which breaks the camel's back.

We have found that children who have underling anxiety or depression are more likely to develop chronic pain [5]. A big issue we find is what we call catastrophizing, a personality trait especially among parents and kids which is rumination and magnifying the problem and constantly being obsessed about the symptoms [6]. Our brain tells us that pain is a bad thing and we are hardwired to be afraid of pain. Put simply, if you break you ankle, the ankle pain informs you to seek medical help and that you could cause tissue damage if you move this body part. In fact in chronic pain the opposite is true, only by moving the body part does the pain resolve. So fear of pain [7] and catastrophizing is a big issue and that is why in our pain clinic we routinely include a family therapist and social worker who are exclusively working with the parents to talk about parenting strategies about how we get their child back to school and reduce parental catastrophizing [2].

Q How have treatment plans at Children's Minnesota evolved over the past 10 years? We really have moved away from the ideas that if a child is living in pain that we basically have to just find a better medication combination or find a better interventional (needle) procedure. In other words, a decade or more ago if there was child with a severe painful condition such as bone cancer or sickle cell crisis we probably would have relied much more heavily on medications alone than we do now. We came to understand 
that many children with severe pathologies also experience chronic pain on top of their underlying condition. And therefore we must utilize both acute pain modalities as well as chronic pain therapies in order to treat them well. We are much less dependent on opioids as the only therapy and much more reliant on psychology/physical therapy, normalizing their schedule in addition to finding the best analgesic medications.

We usually do not have patients on long-term opioids anymore, of course with the exception of recurrent tissue injury, such as children with epidermolysis bullosa or osteogenesis imperfecta, or during their end-of-life period; 10 years ago it was common practice nationwide that some children would be on opioids for years because they had sickle cell disease or an underlying avascular necrosis. This has changed, although of course short-term opioid administration continues to be a basic pillar of state-of-the-art acute pain management. We have now realized that for better pain control we often need to get patients off opioids long term and we need to provide multimodal analgesia [1].

\section{Q In 2016 you received the Elizabeth} Narcessian Award for Outstanding Educational Achievements in the Field of Pain by the American Pain Society. This award recognizes outstanding contributions highlighting dedication or innovation in education in the field of pain. You have given more than 500 lectures in 27 countries on all six continents in the last 15 years: Where do you see your contribution to the field and what role does training \& education play to improve outcomes for children in pain?

In pediatric pain medicine we continue to have an astonishing gap between what we know and what we do, between evidence and practice. Providing excellent pain control often is not rocket science. The evidence for excellent analgesia has been there for many years and we do know how to provide outstanding pain control for children. Both in low-income and in highincome countries the obstacles are commonly very similar. One of the continuing myths hindering the use of opioids in children include unfounded fear about addiction, respiratory depression or 'masking the symptoms'. We have to address the gap in pain treatment of children and education is one of the key tools.

I was lucky enough to secure, with excellent co-investigators, a US\$1.6 million grant from the NIH/National Cancer Centre for Education in Palliative and End-of-Life Care Program (EPEC-Pediatrics) [8] and we were able to develop the most comprehensive curriculum for pediatric pain and palliative care to date. At the heart of this curriculum was the premise that teaching alone does not change behavior of participants (i.e., if someone shows up with a PowerPoint presentation it will most likely not change practice of anyone in the audience). So in my teaching there is a strong emphasis on how we can address attitudes, use knowledge and teach skills to subsequently change behavior and in addition provide information of 'teaching how to teach'.

To give you an example of how I might address attitudes, myths and misconceptions: In every country I went to, I will play out a variation of a case scenario during a lecture, something like "its 3 o'clock in the morning and there is a 10 -yearold child coming into the emergency room in extreme acute pain, a condition you cannot fix right now, and you would expect her to be in pain due to her severe tissue injury for more than $24 \mathrm{~h}$. It crosses your mind to prescribe some morphine. What would your colleagues or the parents say, why you should not prescribe opioids?" I always open that up for discussion, and the answers are always the same, on every continent, whether it is a resource-rich or a resource-poor county: People are afraid of addiction, respiratory depression, inhibiting brain development, causing side effects such as constipation, nausea and itching and some feel that it is culturally inappropriate to mitigate pain. So only by addressing each of these myths in detail during the remainder of my lecture and discussion may I be successful in changing the behavior of the participants in the audience on that day.

So we 'teach participants how to teach', address attitudes and then provide them with the knowledge and then lastly give them a skill. Using little case examples we might invite the audience "Please write the morphine script for this child which weighs $20 \mathrm{~kg}$." After they have written the prescription we might go on: "Now this child becomes nauseous we have to switch from morphine to another strong opioid, how would you do that?" After working on case examples, participants might be much better equipped to address such situation on their own.

Other than EPEC-Pediatrics, through which we have now trained over 400 clinicians worldwide including teams from every single 
Canadian hospital, I am also the course director of the annual pediatric pain masterclass course [9]. This is a 7 days crash course where we cover all areas of multimodal pediatric analgesia. I have the privilege to invite many of the best speakers and content experts in the field of pediatric pain management. We make sure that we address attitudes, provide knowledge and teach skills in many important aspects of pediatric pain management including: simple analgesia, education, opioids and using new anesthesia interventions such as nerve blocks, perivertibal blocks and epidural catheters as well psychology and rehabilitation and how to integrate nonpharmacological modalities.

Most of our participants tell us that after the pain masterclass they are able to deal with roughly $90 \%$ of all pain issues within the children's hospital. At our masterclass we always invite and grant scholarships to international scholars from lower income countries who are able to implement the techniques they learned while we mentor them from far. In addition, we also train junior clinicians and do day-to-day training with nurses and physicians.

Q Is there a specific research area you will be pursuing in the future \& what do you hope to achieve?

Among the issues I am currently most interested in is how we can implement clinical practice change and maintain it. Part of the systemwide Children's Comfort Promise [10] at our institution is focused on how we can change large healthcare systems. We still have a gap of about 17 years before new research reaches the patient. What we are doing right now, such as implementing the Comfort Promise modalities for preventing or decreasing pain caused by needles (including not holding children down, applying numbing cream, using sucrose or breast feeding, and distracting the kids), in fact much of the data and research about this are 20 or more years old. One of the major aims right now is to make what we have achieved here at Children's Minnesota replicable at other institutions. This would help millions of children in North America and worldwide. Through a grant from The Mayday Fund we are now able to implement the Comfort Promise in four children's hospitals in Canada (Toronto, Montreal) and the USA (Kansas City, Atlanta). We also are planning multicenters study to look at how we can use certain quality improvement modalities to change behavior toward improved pain prevention and treatment.

My personal interest is in the ethics behind pain research. I strongly believe that in 2017 it is not appropriate to enroll children into a 'no treatment'-arm in a pain control trial, if clinical evidence exists for safe and efficacious analgesia. In randomized trials of analgesic interventions, infants and children in control groups often do not receive analgesia during painful procedures. From a review of 45 clinical trials (published between 2013 and 2015) of new analgesic treatments for procedural pain in neonates, Bellieni and Johnston found that infants in control groups during painful procedures did not receive analgesia in the majority of cases [11]. Another inappropriate example represents a recent report titled: 'Office Insertion of Tympanostomy Tubes without Anesthesia in Young Children', where Rosenfeld et al. describe using a 'papoose board for restraint' while performing an exquisitely painful procedure, myringotomy and tympanostomy tube insertion without any analgesia or anesthesia [12]. Today it is simply inappropriate and unethical to perform elective painful procedures on children without treatment to avoid or minimize pain [13]. We recently received funding from The Mayday Fund and together with my colleagues Elliot Krane from Stanford, CA, USA, we have gathered a group of national and international experts to develop consensus guidelines for institutional ethics committees to refuse clinical trials that expose infants and children to unnecessary pain, and even more importantly exhorts journal editors not to publish such clinical studies.

\section{Disclaimer}

The opinions expressed in this interview are those of the interviewee and do not necessarily reflect the views of Future Medicine Ltd.

Financial \& competing interests disclosure S Friedrichsdorf is supported, in part, by the Mayday Fund, NIH/National Cancer Institute, Children's Hospitals and Clinics of Minnesota Research Grant Program, NIH/National Institute of Nursing Research and the Canadian Partnership Against Cancer. S Friedrichsdorf has no other relevant affliations or financial involvement with any organization or entity with a financial interest in or financial conflict with the subject matter or materials discussed in the manuscript apart from those disclosed.

No writing assistance was utilized in the production of this manuscript. 


\section{References}

Papers of special note have been highlighted as: - of interest; $\bullet \bullet$ of considerable interest

1 Friedrichsdorf SJ. Prevention and Treatment of Pain in Hospitalized Infants, Children, and Teenagers: From Myths and Morphine to Multimodal Analgesia. Pain 2016: Refresher Courses 16th World Congress on Pain. International Association for the Study of Pain, IASP Press, Washington, DC, USA, 309-319 (2016).

2 Friedrichsdorf SJ, Giordano J, Desai-Dakoji $\mathrm{K}$, Warmuth A, Schulz CA. Chronic Pain in children and adolescents: diagnosis and treatment of primary pain disorders in head, abdomen, muscles and joints. Children (Basel) 3(4), E42 (2016).

3 King S, Chambers CT, Huguet A et al. The epidemiology of chronic pain in children and adolescents revisited: a systematic review. Pain 152(12), 2729-2738 (2011).

Huguet A, Miro J. The severity of chronic pediatric pain: an epidemiological study. J. Pain 9(3), 226-236 (2008).
5 Tegethoff M,Belardi A, Stalujanis E, Meinlschmidt G. Comorbidity of mental disorders and chronic pain: chronology of onset in adolescents of a national representative cohort. J. Pain 16(10), 1054-1064 (2015).

6 Lynch-Jordan AM, Kashikar-Zuck S, Szabova A, Goldschneider KR. The interplay of parent and adolescent catastrophizing and its impact on adolescents' pain, functioning, and pain behavior. Clin. J. Pain 29, 681-688 (2013).

7 Simons LE, Kaczynski KJ, Conroy C, Logan DE. Fear of pain in the context of intensive pain rehabilitation among children and adolescents with neuropathic pain: associations with treatment response. J. Pain 13(12), 1151-1161 (2012).

8 Education in Palliative and End-of-Life Care: Pediatrics.

http://bioethics.northwestern.edu

9 Department of Pain Medicine, Palliative Care $\&$ Integrative Medicine: 10th Annual
Pediatric Pain Master Class. http://noneedlesspain.org/ppmc

10 Friedrichsdorf SJ, Eull D, Weidner CA. Children's Comfort Promise: how can we do everything possible to prevent and treat pain in children using quality improvement strategies? (commentary). Pediatric Pain Letter 18(3), 26-30 (2016).

11 Bellieni CV, Johnston CC. Analgesia, nil or placebo to babies, in trials that test new analgesic treatments for procedural pain. Acta Paediatr. 105(2), 129-136 (2016).

12 Rosenfeld RM, Sury K, Mascarinas C. Office insertion of tympanostomy tubes without anesthesia in young children. Otolaryngol. Head Neck Surg. 153(6), 1067-1070 (2015).

13 Friedrichsdorf SJ, Sidman J, Krane EJ. Prevention and treatment of pain in children: toward a paradigm shift. Otolaryngol. Head Neck Surg. 154(5), 804-805 (2016) 